\title{
Association between dipeptidyl peptidase-4 inhibitors and risk of bullous pemphigoid in patients with diabetes: A population-based cohort study
}

Chen-YiWu ( $\nabla$ chenyiok@gmail.com )

Taipei Veterans General Hospital https://orcid.org/0000-0002-8393-3900

Chun-Ying Wu

National Yang-Ming University

Chung-Pin Li

Taipei Veterans General Hospital

Yiing-Jenq Chou

National Yang-Ming University

Yi-Hsian Lin

National Yang Ming University

Yun-Ting Chang

Taipei Veterans General Hospital

Original investigation

Keywords: dipeptidyl peptidase 4 inhibitor, bullous pemphigoid, cohort study, risk factor

Posted Date: July 8th, 2020

DOI: https://doi.org/10.21203/rs.3.rs-38822/v1

License: (9) (i) This work is licensed under a Creative Commons Attribution 4.0 International License.

Read Full License 


\section{Abstract \\ Background}

Higher bullous pemphigoid (BP) risk has been reported to be associated with dipeptidyl peptidase 4 inhibitor (DPP4i). However, large-scale studies to investigate the association between BP and DPP4i treatment are limited. The aim of this study is to examine the association between BP risk and DPP4i treatment in diabetes patients.

\section{Methods}

We conducted a nationwide cohort study based on the Taiwan National Health Insurance Database between 2000 and 2015. 124,619 diabetic patients who were receiving DPP4i therapy were matched 1: 1 with diabetic patients who had never received DPP4i by age, sex, duration of diabetes, insulin usage, and propensity score-matching of comorbidities.

\section{Results}

Of the 124,619 diabetes patients in the two groups, the mean age at diabetes diagnosis was $52.4 \pm 10.9$ years, with a mean duration of diabetes of $6.0 \pm 3.9$ years. After adjusting for competing mortality risk, the 6-year cumulative incidence of BP in the DPP4i-treated cohort was significantly higher than that in the non-DPP4i group (0.74 per 1000 ; $95 \%$ confidence interval [Cl]: $0.51-1.05$ vs 0.38 per 1000 ; $95 \%$ Cl: $0.26-$ $0.53, P=.001)$. The DPP4i and insulin-treated group had the highest 6 -year cumulative incidence for BP (0.93; 95\% Cl: $0.54-1.54$ per 1000). Modified Cox regression analysis revealed that DPP4i treatment (HR: 2.15, 95\% Cl: 1.18-3.91, $\mathrm{P}=0.01)$, age (HR: 1.06, $\mathrm{P}<.001$ ), renal disease (HR: 2.32, $\mathrm{P}<.001)$, and metformin user (HR: 1.93, $P=0.006)$ were associated with increased $B P$ risk.

\section{Conclusion}

DPP4i users had a 2.2-fold increase in the risk of BP, and the risk was the highest in those with concomitant use of DPP4i and insulin. The use of DPP4is as anti-diabetic medications must be monitored carefully and may be replaced by other anti-diabetic medications in BP patients.

\section{Background}

Bullous pemphigoid (BP) is a common autoimmune blistering skin disease associated with substantial morbidity and mortality $[1,2]$. The incidence of BP has been reported to be approximately 13.4-66 new cases per 1 million people per year [3, 4]. BP is accompanied by intense pruritus and can cause considerable disability and mortality, typically affecting elderly patients $[2,5,6]$. A recent meta-analysis reported a 1-year mortality rate of $23.5 \%$ in patients with BP [7]. BP is characterized by autoantibodies 
directed against hemidesmosomal proteins (BP180 and BP230) at the dermoepidermal junction [8]. A 1.9to 4.3-fold increase in the incidence of BP has been reported in the past two decades $[3,4,9]$. One possible explanation is the increased use of some medications in older adults [3].

Dipeptidyl peptidase-4 inhibitors (DPP4is) are a new class of oral antidiabetic agents for patients with type 2 diabetes [10]. Recent studies have suggested that DPP4is are associated with an increased risk of BP [11-23]. However, there were some limitations in previous studies, for example, advanced and severe diabetes patients were excluded [23] or the duration of diabetes and comorbidities were not well controlled $[11,12,22]$. The interaction of DPP4is and insulin on the risk of BP has never been investigated. The temporal relationship between the use of DPP4is and BP is unclear. Furthermore, the association between sex and age of the DPP4i-treated group with the development of BP remains controversial $[12,17,21]$. Large-scale cohort studies are warranted to further increase the knowledge in these areas.

Therefore, we conducted a nationwide population-based cohort study to investigate the use of DPP4is and risk of BP.

\section{Methods}

\section{Data source}

Data used in this nationwide cohort study were obtained from the National Health Insurance Research Database (NHIRD) in Taiwan since January 1, 2000, to December 31, 2015. The NHIRD was set up in 1997 from the National Health Insurance (NHI), which is a mandatory national health insurance program that covers more than $99 \%$ of the Taiwanese population [24]. The database contains comprehensive information, including demographic characteristics, disease diagnoses, and details of prescriptions. Diseases were recorded using the International Classification of Diseases, Ninth Revision, Clinical Modification (ICD-9-CM). The NHIRD has been widely used in epidemiological studies and is one of the most complete health care service databases in the world [25]. The quality of the NHIRD, in terms of the diagnostic accuracy of major diseases and medications, has been well validated [26, 27]. Data confidentiality is assured by the regulations of the NHIRD Administration, Ministry of Health and Welfare, Taiwan. This study was performed in accordance with the Helsinki Declaration and was approved by the National Health Research Institutes and the Institutional Review Board of Taipei Veterans General Hospital (IRB No.: 2019-01-007AC).

\section{Study population}

The patient selection process is shown in Figure 1. Diabetic patients were evaluated between January 1 , 2000, and December 31, 2015. Individuals were defined as having diabetes if there were at least three diagnosis codes (ICD-9-CM: 250) in their outpatient clinic records or one in their admission record, and if they had at least 3-months of anti-diabetic medications. To avoid confounding and to investigate the impact of DPP4i on BP, we excluded diabetic patients with cancers and neurological diseases, including 
cerebrovascular diseases, dementia, Alzheimer's disease, Parkinson's disease, epilepsy, multiple sclerosis, and psychiatric diseases, which are well-known risk factors for BP [28, 29].

Sitagliptin was the first DPP4i reimbursement by NHI in Taiwan in 2009, followed by saxagliptin and vidagliptin in 2011, and linagliptin in 2012. They were used alone or in combination with metformin as the second line treatment for diabetes. Since no data is available for the duration of DPP4i use to the risk of BP, a selection criterion of 3 months was used for enrolling DPP4i users. Patients who had used DPP4 $i \geq 28$ days per month for at least 3 months were identified as the DPP4i-treated group. An index date was assigned as the date after 3 months of DPP4i medication. The duration of diabetes was calculated from the date of diabetes diagnosis to the index date. We defined those who had used metformin at least 3 months over 1 year as metformin user. To further identify severely diabetic patients who required insulin treatment, insulin users were defined as diabetic patients who had used insulin for at least 3 months over 1 year.

Patients who used other antidiabetic medications, with the exception of DPP4is, for at least 3 months were assigned to the non-DPP4i-treated cohort. Because DPP4is were used as a second line anti-diabetic medication, those who had not taken DPP4is may have been new-onset or mild diabetic patients. Because diabetes has been reported as a risk factor for BP [30], we selected patients with the same duration of diabetes to include a comparable control. We assigned a pseudo-index date for each nonDPP4i patient and included those with the same duration of diabetes with the DPP4i-treated group. Patients who met the exclusion criteria before the pseudo-index date of non-DPP4i group were further excluded. A total of 124,619 patients who received DPP4i therapy were randomly matched 1:1 with those who had never received DPP4i by means of age ( \pm 0.5 year), sex, duration of diabetes ( \pm 0.5 year), insulin usage, and propensity scores $( \pm 0.01)$ of comorbidities.

\section{Outcome measurement}

The occurrence of BP was the measured outcome. Patients who were diagnosed with BP were identified by ICD-9-CM 694.5. Individuals were defined as having BP if there were at least three diagnosis codes in their outpatient clinic records or one in admission record. Patients who developed BP before the index dates were excluded. Study participants were followed up until the date of BP diagnosis, death, or the end of the study period.

\section{Assessment of accuracy of BP identification algorithm}

To evaluate the accuracy of BP diagnosis by our defined algorithm, we retrieved BP data from the electronic medical records of the Taipei Veterans General Hospital (a 2,802-bed teaching hospital in Taipei) between January 1, 2011 and December 31, 2015. We reviewed patients' clinical information, pathology results, direct and indirect immunoflourescence data, and medical records, as reference standards to estimate the positive predictive value of the BP identification algorithm. According to the BP identification algorithm, a total of 247 patients with BP were identified from the electronic medical records of the Taipei Veterans General Hospital. Among these algorithm-identified patients, 242 were 
confirmed through medical record reviews, suggesting that our algorithm had a good positive prediction value of $98.0 \%$ (95\% confidence interval [CI]: $96.3-99.7 \%)$

\section{Major coexisting diseases}

Several major coexisting diseases that might be associated with the risk of BP were identified by ICD-9$\mathrm{CM}$ codes and included coronary artery disease, hypertension, hyperlipidemia, renal disease, chronic liver disease and cirrhosis, chronic obstructive pulmonary disease, and connective tissue disease. The diseases were included in the calculation of propensity score.

\section{Statistical analysis}

Continuous data were summarized with mean, median and interquartile range (IQR) whereas categorical data with number and proportion, unless otherwise specified. Cumulative incidence rates of BP during the follow-up were calculated. After adjusting for competing mortality, cumulative incidence rates were calculated and compared using modified Kaplan-Meier and Gray methods [25]. A modified log-rank test was used to compare differences in the full time-to-event distribution between the DPP4i-treated and untreated groups.

Multivariable analyses using hazard ratios (HRs) were performed with modified Cox hazards models in the presence of competing risk events, and adjusted for covariate factors, including use of DPP4i, age, sex, duration of diabetes, all the major coexisting diseases, use of insulin, use of metformin, and the interaction term of DPP4i and insulin. Further stratified multivariable analyses were performed. Two-sided $P$ values $<.05$ were considered statistically significant. All data were managed using SAS software, version 9.3 (SAS Institute, Gary, NC). The Cox proportional hazard regression models in the competing risk analysis were carried out using the "cmprsk" package of R (http://cran.rproject.org/web/packages/cmprsk/index.html).

\section{Results}

\section{Characteristic of the study subjects}

After 1:1 matching of the DPP4i-treated and non-DPP4i-treated groups, a total of 249,238 diabetic patients were included (Table I). Of them, there were 44 BP patients in the DPP4i-treated group, and 47 BP patients in the non-DPP4i-treated group. The mean follow-up time was significantly shorter in the DPP4itreated group ( $3.2 \pm 1.8$ vs $5.5 \pm 3.8$ years for DPP4i-treated and non-DPP4i-treated group, respectively). There were $80,185(64.3 \%)$ men and 44,434 (35.7\%) women in each group, with a mean ages of diabetes diagnosis of $52.4 \pm 10.9$ years and a mean duration of diabetes ( $6.0 \pm 3.9$ years). There were no significant differences in the propensity scores of their comorbidities between these two groups.

\section{Six-year cumulative incidences of BP}


After adjusting for competing mortality, patients in the DPP4i-treated group had a significantly higher 6year cumulative incidence of BP than the non-DPP4i-treated group $(0.74 ; 95 \% \mathrm{Cl}$ : $0.51-1.05$ vs $0.38 ; 95 \%$ Cl: $0.26-0.53$ per $1000, P=.001$ ) (Figure 2). The DPP4i and insulin-treated group had the highest 6-year cumulative incidence for BP $(0.93 ; 95 \% \mathrm{Cl}$ : $0.54-1.54$ per 1000), followed by the DPP4i and non-insulintreated group ( $0.64 ; 95 \% \mathrm{Cl}: 0.39-1.03$ per 1000$)$, the non-DPP4i and insulin-treated group $(0.50 ; 95 \% \mathrm{Cl}$ : $0.29-0.84$ per 1000$)$, and the non-DPP4i and non-insulin-treated group $(0.31 ; 95 \% \mathrm{Cl}: 0.19-0.50$ per 1000).

\section{Multivariable analysis of risk factors}

In multivariable regression analysis adjusted for competing mortality and covariates, the use of DPP4i was an independent risk factor for BP (HR: 2.15, 95\% Cl: 1.18-3.91, P = 0.01) (Table II). Age (HR: 1.06), renal disease (HR: 2.32), and metformin user (HR: 1.93) were associated with increased risk of BP.

\section{Multivariable stratified analysis for use of DPP4i}

Multivariable stratified analysis was performed for subgroups of patients and adjusted for the same covariates as the previous modified Cox model, with the exception of the interaction term of DPP4i and insulin (Figure 3). In the subgroup analysis, the risk of BP significantly increased in some DPP4i-treated subgroups, including in patients aged 60-69 (HR: 3.40), male sex (HR: 2.43), without coronary artery disease (HR: 1.97), without hypertension (HR: 5.33), with hyperlipidemia (HR: 2.32), without renal disease (HR: 2.34), without chronic liver disease and cirrhosis (HR: 2.00), without chronic obstructive pulmonary disease (HR: 2.17), without connective tissue disease (HR:1.98), non-insulin users (HR: 2.26), and nonmetformin user (HR:2.09). The overall risk of BP was 1.75-fold higher (95\% Cl: 1.14-2.67) in the DPP4itreated group.

\section{Discussion}

This nationwide population-based cohort study showed that the DPP4i was associated with a 2.2-fold increase in the risk of BP. Without adjustment for the interaction term of DPP4i and insulin, DPP4i treatment remained a 1.8-fold increase in the risk of BP. Previous studies have reported that DPP4is increased the risk of BP by 1.6 to 3.2 -fold [11, 12, 21-23]. However, these studies might have some methodological weaknesses, such as potential selection bias [21, 31], hospital setting [11, 22], excluding advanced diabetic patients using insulin [23], or not controlling for the duration of diabetes [12], that might have complicated the interpretation of their findings. In our study, we excluded major confounders and chose non-DPP4i diabetic patients with the same diabetic duration and propensity score-matching of comorbidities. Thus, the magnitude for the risk of DPP4is for BP is expected to be more accurate.

The duration of diabetes might be a risk factor for BP. When lifestyle intervention and oral therapies are no longer fully successful in diabetic control, insulin is usually the most desirable therapy [32]. As DPP4is are used as a second line of anti-diabetic treatment for advanced diabetes, when investigating the association of DPP4i and BP, it is important to control for the diabetic duration and use of insulin. We 
observed the highest risk in those with concomitant use of DPP4i and insulin. These results imply that the impact of DPP4is on the development of BP in diabetic patients might be additive with insulin, which was a proxy of diabetic severity. Metformin user was observed to increase the risk of BP in our study. However, the association of DPP4i use with BP was independent of the use of metformin.

The association of sex and risk of BP in the DPP4i-treated group remained conflicting. Garcia et al. observed that the development of BP in diabetes patients was more common in men [17]. Lee et al. and Benzaquen et al. reported that DPP4is were associated with an increased risk of BP in men but not in women with diabetes $[12,22]$. Kridin et al. revealed that the association of DPP4i with BP was stronger among diabetic men [11]. However, Varpuluoma et al. observed that women were more likely than men to develop BP after DPP4i intake [21]. In our study, the risks of BP associated with DPP4is were significantly increased in men but not in women.

The association between age and increased risk of BP in the DPP4i-treated group showed inconsistent results. Benzaquen et al. has reported that DPP4i treatment was strongly associated with BP in patients aged $>80$ years [22]. Kridin et al. observed that the risk was the strongest in patients aged $<70$ years [11]. Lee et al. presented that the risk was slightly higher in those aged $<75$ years [12]. In our study, the risk of BP associated with DPP4i was only significantly increased in patients aged 60-69 years.

The median latent period between the initiation of DPP4i treatment and diagnosis of BP varied among different studies, ranging from 5 months to over 4 years $[11,18,19,21,22,31,33]$. In our study, the median latent time was 2.5 years, with a minimum of 73 days to a maximum of 6.2 years (quartiles 1-3: 1.3-4.1 years). The long latent period between DPP4i treatment and the initiation of BP suggests that it is a drug-aggravated condition rather than a drug-induced cutaneous reaction.

The exact mechanism of how DPP4i induces BP remains unclear. DDP4 is a cell surface plasminogen receptor that can activate plasminogen and lead to plasmin formation [34]. Plasmin is a major serine protease that cleaves BP180 within the immunodominant NC16A domain [35]. DPP4i can inhibit plasmin and may then alter the proper cleavage of BP180, which may affect its antigenicity and function [36]. It has been previously reported that DPP4i might alter the antigenic properties of the epidermal basement membrane and lead to modifications of the immune response in predisposed patients $[16,37,38]$.

The study has several strengths. First, this study was a nationwide population-based study that included large numbers of diabetes patients. We could evaluate the association between DPP4i and BP, and further subgroup analyses. Second, to scrutinize the effects of DPP4i on BP, we excluded diabetic patients with neurological diseases that were significant risk factors for BP. Third, as an increase in the duration and severity of diabetes were risk factors for BP, we chose non-DPP4i-treated diabetic patients with the same diabetic duration and percentage of insulin users as the control group. Our findings provide solid epidemiological evidence that DPP4is are associated with an increased risk of BP.

The potential limitations of our study should also be considered. First, as an observational study, residual confounding remains possible. We attempted to avoid residual confounding by choosing good controls. 
Second, administrative data are subject to coding errors. However, these coding errors belong to nondifferential misclassification bias that would make our results more conservative. Third, because using of administrative data, we have no access to information of the severity or clinical characteristics of diabetes or BP. We used insulin usage as a proxy for diabetic severity. Finally, because almost all of our subjects were Taiwanese, our results may not be generalizable to other ethnic groups.

\section{Conclusion}

Our study revealed that DPP4is were associated with a 2.2-fold increase in the risk of BP, and the risk was the highest in those using DPP4is and insulin concomitantly. The use of DPP4is as anti-diabetic medications must be monitored carefully and may be replaced by other anti-diabetic medications in BP patients.

\section{Abbreviations}

DPP4i: Dipeptidyl peptidase 4 inhibitor; BP: Bullous pemphigoid; NHIRD: National Health Insurance Research Database; NHI: National Health Insurance; ICD-9-CM: International Classification of Diseases, Ninth Revision, Clinical Modification; Cl: Confidence interval; IQR: Interquartile range; HR: Hazard ratio.

\section{Declarations}

\section{Ethics approval}

This study is approved by the National Health Research Institutes and the Institutional Review Board of Taipei Veterans General Hospital (IRB No.: 2019-01-007AC).

\section{Acknowledgments}

This study was supported by grants from the Ministry of Science and Technology, R.O.C. (MOST 1082314-B-075-041-MY3).

\section{Availability of data and materials}

The datasets generated and analyzed during the current study are available in the National Health Insurance Research Database. https://dep.mohw.gov.tw/dos/np-2497-113.html.

\section{Competing interests}

The authors declare that they have no competing interests.

\section{Funding}

This study was supported by grants from the Ministry of Science and Technology, Taiwan, R.O.C. (MOST 108-2314-B-075-041-MY3). 


\section{Authors' contributions}

All the authors took part in the design of the study and approved the final version of the manuscript to be published. Chen-Yi W and CPL researched data and wrote the manuscript. Chen-Yi W and Chun-Ying W researched data and reviewed/edited the manuscript. YJC, YHL and YTC contributed to the discussion and reviewed the manuscript. Chen-Yi W is the guarantor of this work.

\section{Consent for publication}

Not applicable

\section{References}

1. Ren Z, Hsu DY, Brieva J, Silverberg NB, Langan SM, Silverberg JI. Hospitalization, inpatient burden and comorbidities associated with bullous pemphigoid in the U.S.A. Br J Dermatol. 2017;176(1):8799.

2. Schmidt E, Zillikens D. Pemphigoid diseases. Lancet. 2013;381(9863):320-32.

3. Joly P, Baricault S, Sparsa A, Bernard P, Bedane C, Duvert-Lehembre S, Courville P, Bravard P, Remond $B$, Doffoel-Hantz V, et al. Incidence and mortality of bullous pemphigoid in France. J Invest Dermatol. 2012;132(8):1998-2004.

4. Langan SM, Smeeth L, Hubbard R, Fleming KM, Smith CJ, West J. Bullous pemphigoid and pemphigus vulgaris-incidence and mortality in the UK: population based cohort study. BMJ. 2008;337:a180.

5. Marazza G, Pham HC, Scharer L, Pedrazzetti PP, Hunziker T, Trueb RM, Hohl D, Itin P, Lautenschlager $S$, Naldi L, et al. Incidence of bullous pemphigoid and pemphigus in Switzerland: a 2-year prospective study. Br J Dermatol. 2009;161(4):861-8.

6. Jung M, Kippes W, Messer G, Zillikens D, Rzany B. Increased risk of bullous pemphigoid in male and very old patients: A population-based study on incidence. J Am Acad Dermatol. 1999;41(2 Pt 1):2668.

7. Kridin K, Shihade W, Bergman R. Mortality in Patients with Bullous Pemphigoid: A Retrospective Cohort Study, Systematic Review and Meta-analysis. Acta Derm Venereol. 2019;99(1):72-7.

8. Kasperkiewicz M, Zillikens D. The pathophysiology of bullous pemphigoid. Clin Rev Allergy Immunol. 2007;33(1-2):67-77.

9. Kridin K, Bergman R. Ethnic variations in the epidemiology of bullous pemphigoid in Israel. Int J Dermatol. 2018;57(1):34-9.

10. Thornberry NA, Gallwitz B. Mechanism of action of inhibitors of dipeptidyl-peptidase-4 (DPP-4). Best Pract Res Clin Endocrinol Metab. 2009;23(4):479-86.

11. Kridin K, Bergman R. Association of Bullous Pemphigoid With Dipeptidyl-Peptidase 4 Inhibitors in Patients With Diabetes: Estimating the Risk of the New Agents and Characterizing the Patients. 
JAMA Dermatol. 2018;154(10):1152-8.

12. Lee SG, Lee HJ, Yoon MS, Kim DH. Association of Dipeptidyl Peptidase 4 Inhibitor Use With Risk of Bullous Pemphigoid in Patients With Diabetes. JAMA Dermatol. 2019;155(2):172-7.

13. Haber R, Fayad AM, Stephan F, Obeid G, Tomb R. Bullous Pemphigoid Associated With Linagliptin Treatment. JAMA Dermatol. 2016;152(2):224-6.

14. Mendonca FM, Martin-Gutierrez FJ, Rios-Martin JJ, Camacho-Martinez F. Three Cases of Bullous Pemphigoid Associated with Dipeptidyl Peptidase-4 Inhibitors - One due to Linagliptin. Dermatology. 2016;232(2):249-53.

15. Aouidad I, Fite C, Marinho E, Deschamps L, Crickx B, Descamps V. A case report of bullous pemphigoid induced by dipeptidyl peptidase-4 inhibitors. JAMA Dermatol. 2013;149(2):243-5.

16. Skandalis K, Spirova M, Gaitanis G, Tsartsarakis A, Bassukas ID. Drug-induced bullous pemphigoid in diabetes mellitus patients receiving dipeptidyl peptidase-IV inhibitors plus metformin. J Eur Acad Dermatol Venereol. 2012;26(2):249-53.

17. Garcia M, Aranburu MA, Palacios-Zabalza I, Lertxundi U, Aguirre C. Dipeptidyl peptidase-IV inhibitors induced bullous pemphigoid: a case report and analysis of cases reported in the European pharmacovigilance database. J Clin Pharm Ther. 2016;41(3):368-70.

18. Bene J, Moulis G, Bennani I, Auffret M, Coupe P, Babai S, Hillaire-Buys D, Micallef J, Gautier S. French Association of Regional PharmacoVigilance C: Bullous pemphigoid and dipeptidyl peptidase IV inhibitors: a case-noncase study in the French Pharmacovigilance Database. Br J Dermatol. 2016;175(2):296-301.

19. Lindgren O, Varpuluoma O, Tuusa J, Ilonen J, Huilaja L, Kokkonen N, Tasanen K. Gliptin-associated Bullous Pemphigoid and the Expression of Dipeptidyl Peptidase-4/CD26 in Bullous Pemphigoid. Acta Derm Venereol. 2019;99(6):602-9.

20. Plaquevent M, Tetart F, Fardet L, Ingen-Housz-Oro S, Valeyrie-Allanore L, Bernard P, Hebert V, Roussel A, Avenel-Audran M, Chaby G, et al. Higher Frequency of Dipeptidyl Peptidase-4 Inhibitor Intake in Bullous Pemphigoid Patients than in the French General Population. J Invest Dermatol. 2019;139(4):835-41.

21. Varpuluoma O, Forsti AK, Jokelainen J, Turpeinen M, Timonen M, Huilaja L, Tasanen K. Vildagliptin Significantly Increases the Risk of Bullous Pemphigoid: A Finnish Nationwide Registry Study. J Invest Dermatol. 2018;138(7):1659-61.

22. Benzaquen M, Borradori L, Berbis P, Cazzaniga S, Valero R, Richard MA, Feldmeyer L. Dipeptidyl peptidase IV inhibitors, a risk factor for bullous pemphigoid: Retrospective multicenter case-control study from France and Switzerland. J Am Acad Dermatol. 2018;78(6):1090-6.

23. Douros A, Rouette J, Yin H, Yu OHY, Filion KB, Azoulay L. Dipeptidyl Peptidase 4 Inhibitors and the Risk of Bullous Pemphigoid Among Patients With Type 2 Diabetes. Diabetes Care. 2019;42(8):1496503.

24. Rachel Lu JF, Chiang TL. Evolution of Taiwan's health care system. Health Econ Policy Law. 2011;6(1):85-107. 
25. Wu CY, Chen YJ, Ho HJ, Hsu YC, Kuo KN, Wu MS, Lin JT. Association between nucleoside analogues and risk of hepatitis $B$ virus-related hepatocellular carcinoma recurrence following liver resection. JAMA. 2012;308(18):1906-14.

26. Cheng CL, Kao YH, Lin SJ, Lee CH, Lai ML. Validation of the National Health Insurance Research Database with ischemic stroke cases in Taiwan. Pharmacoepidemiol Drug Saf. 2011;20(3):236-42.

27. Cheng $\mathrm{CL}$, Lee $\mathrm{CH}$, Chen PS, Li YH, Lin SJ, Yang YH. Validation of acute myocardial infarction cases in the national health insurance research database in taiwan. J Epidemiol. 2014;24(6):500-7.

28. Milani-Nejad N, Zhang M, Kaffenberger J. The association between bullous pemphigoid and neurological disorders: a systematic review. Eur J Dermatol. 2017;27(5):472-81.

29. Wu CY, Hu HY, Chou YJ, Li CP, Chang YT. Neurological Cancer is a Risk Factor for Bullous Pemphigoid: 11-Year Population-Based Cohort Study. Am J Clin Dermatol 2019.

30. Chen CT, Hu HY, Chang YT, Li CP, Wu CY. Cancer is not a risk factor for bullous pemphigoid: 10-year population-based cohort study. Br J Dermatol. 2019;180(3):553-8.

31. Schaffer C, Buclin T, Jornayvaz FR, Cazzaniga S, Borradori L, Gilliet M, Feldmeyer L. Use of Dipeptidyl-Peptidase IV Inhibitors and Bullous Pemphigoid. Dermatology. 2017;233(5):401-3.

32. Home P, Riddle M, Cefalu WT, Bailey CJ, Bretzel RG, Del Prato S, Leroith D, Schernthaner G, van Gaal L, Raz I. Insulin therapy in people with type 2 diabetes: opportunities and challenges? Diabetes Care. 2014;37(6):1499-508.

33. Chijiwa C, Takeoka S, Kamata M, Tateishi M, Fukaya S, Hayashi K, Fukuyasu A, Tanaka T, Ishikawa T, Ohnishi T, et al. Decrease in eosinophils infiltrating into the skin of patients with dipeptidyl peptidase4 inhibitor-related bullous pemphigoid. J Dermatol. 2018;45(5):596-9.

34. Gonzalez-Gronow M, Kaczowka S, Gawdi G, Pizzo SV. Dipeptidyl peptidase IV (DPP IV/CD26) is a cell-surface plasminogen receptor. Front Biosci. 2008;13:1610-8.

35. Hofmann SC, Voith U, Schonau V, Sorokin L, Bruckner-Tuderman L, Franzke CW. Plasmin plays a role in the in vitro generation of the linear IgA dermatosis antigen LADB97. J Invest Dermatol. 2009;129(7):1730-9.

36. Izumi K, Nishie W, Mai Y, Wada M, Natsuga K, Ujiie H, Iwata H, Yamagami J, Shimizu H. Autoantibody Profile Differentiates between Inflammatory and Noninflammatory Bullous Pemphigoid. J Invest Dermatol. 2016;136(11):2201-10.

37. Forssmann U, Stoetzer C, Stephan M, Kruschinski C, Skripuletz T, Schade J, Schmiedl A, Pabst R, Wagner L, Hoffmann T, et al. Inhibition of CD26/dipeptidyl peptidase IV enhances CCL11/eotaxinmediated recruitment of eosinophils in vivo. J Immunol. 2008;181(2):1120-7.

38. Klemann C, Wagner L, Stephan M, von Horsten S. Cut to the chase: a review of CD26/dipeptidyl peptidase-4's (DPP4) entanglement in the immune system. Clin Exp Immunol. 2016;185(1):1-21.

\section{Tables}




\begin{tabular}{|c|c|c|c|}
\hline & DPP4i-treated & Non-DPP4i-treated & $P$ value \\
\hline ?s & 124,619 & 124,619 & \\
\hline ihigoid (no, \%) & $44(0.0)$ & $47(0.0)$ & 0.83 \\
\hline \multicolumn{4}{|l|}{ xe (year) } \\
\hline ) & $3.2 \pm 1.8$ & $5.5 \pm 3.8$ & $<0.001$ \\
\hline 1-Q3) & $3.2(1.7-4.6)$ & $4.8(2.4-7.9)$ & $<0.001$ \\
\hline ) & $80,185(64.3)$ & 80,185 (64.3) & $>0.999$ \\
\hline , \%) & $44,434(35.7)$ & $44,434(35.7)$ & $>0.999$ \\
\hline \multicolumn{4}{|l|}{ es diagnosis (year) } \\
\hline$)$ & $52.4 \pm 10.9$ & $52.4 \pm 10.9$ & 0.98 \\
\hline 1-Q3) & $52.7(45.7-59.6)$ & $52.7(45.7-59.6)$ & 0.98 \\
\hline \multicolumn{4}{|l|}{ Date (year) } \\
\hline ) & $58.4 \pm 11.3$ & $58.4 \pm 11.3$ & 0.84 \\
\hline 1-Q3) & 58.9 (51.6-65.7) & 58.9 (51.6-65.7) & 0.83 \\
\hline \multicolumn{4}{|l|}{ liabetes (year) } \\
\hline$)$ & $6.0 \pm 3.9$ & $6.0 \pm 3.9$ & 0.56 \\
\hline 1-Q3) & $5.9(2.4-9.4)$ & $5.9(2.4-9.4)$ & 0.54 \\
\hline \multicolumn{4}{|l|}{ s (no, \%) } \\
\hline artery disease & $30,493(24.5)$ & 31,591 (25.4) & $<0.001$ \\
\hline ion & 89,145 (71.5) & $90,916(73.0)$ & $<0.001$ \\
\hline emia & $97,162(78.0)$ & 96,817 (77.7) & 0.10 \\
\hline ase & $19,224(15.4)$ & $20,168(16.2)$ & $<0.001$ \\
\hline ter diseases and cirrhosis & $34,481(27.7)$ & $37,092(29.8)$ & $<0.001$ \\
\hline sstructive pulmonary disease & $23,011(18.5)$ & $23,927(19.2)$ & $<0.001$ \\
\hline e tissue disease & $3,476(2.8)$ & $3,508(2.8)$ & 0.71 \\
\hline Score $($ Mean \pm SD) & $0.4 \pm 0.1$ & $0.4 \pm 0.1$ & 0.71 \\
\hline Score (Median (Q1-Q3) & $0.4(0.4-0.5)$ & $0.4(0.4-0.5)$ & 0.51 \\
\hline no, \%) & $36,180(29.0)$ & $36,180(29.0)$ & $>0.999$ \\
\hline ser (no, \%) & 34,801 (27.9) & 30,367 (24.4) & $<.001$ \\
\hline
\end{tabular}

Abbreviations: DPP4i: dipeptidyl peptidase-4 inhibitors; SD: standard deviation; IQR: interquartile range

Table 2. Risk of BP after adjusting for competing mortality 


\begin{tabular}{|c|c|c|c|c|}
\hline \multirow[t]{2}{*}{ Variable } & \multicolumn{2}{|c|}{ Numbers BP numbers } & \multicolumn{2}{|c|}{ Multivariate $^{\mathrm{a}}$} \\
\hline & & & HR (95\% CI) & P Value \\
\hline \multicolumn{5}{|l|}{ DPP4i vs Non-DPP4i } \\
\hline Non-treated & 124,619 & 47 & 1 (Ref.) & \\
\hline Treated & 124,619 & 44 & $2.15(1.18-3.91)$ & 0.01 \\
\hline \multicolumn{5}{|l|}{ Sex } \\
\hline Women & 88,868 & 35 & 1 (Ref.) & \\
\hline Men & 160,370 & 56 & $1.17(0.77-1.79)$ & 0.46 \\
\hline Age per year & & & $1.06(1.04-1.09)$ & $<0.001$ \\
\hline Duration of diabetes per year & & & $1.06(1.00-1.12)$ & 0.05 \\
\hline \multicolumn{5}{|l|}{ Coronary artery disease } \\
\hline No & 187,154 & 66 & 1 (Ref.) & \\
\hline Yes & 62,084 & 25 & $0.60(0.37-0.96)$ & 0.03 \\
\hline \multicolumn{5}{|l|}{ Hypertension } \\
\hline No & 69,177 & 14 & 1 (Ref.) & \\
\hline Yes & 180,061 & 77 & $1.13(0.63-2.03)$ & 0.68 \\
\hline \multicolumn{5}{|l|}{ Hyperlipidemia } \\
\hline No & 55,259 & 29 & 1 (Ref.) & \\
\hline Yes & 193,979 & 62 & $0.78(0.50-1.24)$ & 0.30 \\
\hline \multicolumn{5}{|l|}{ Renal Disease } \\
\hline No & 209,846 & 56 & 1 (Ref.) & \\
\hline Yes & 39,392 & 35 & $2.32(1.46-3.68)$ & $<.001$ \\
\hline \multicolumn{5}{|c|}{ Chronic liver disease and cirrhosis } \\
\hline No & 177,665 & 70 & 1 (Ref.) & \\
\hline Yes & 71,573 & 21 & $0.74(0.45-1.22)$ & 0.24 \\
\hline \multicolumn{5}{|c|}{ Chronic obstructive pulmonary disease } \\
\hline No & 202,300 & 62 & 1 (Ref.) & \\
\hline Yes & 46,938 & 29 & $1.25(0.77-2.02)$ & 0.37 \\
\hline \multicolumn{5}{|l|}{ Connective tissue disease } \\
\hline No & 242,254 & 87 & 1 (Ref.) & \\
\hline Yes & 6,984 & 4 & $1.14(0.41-3.14)$ & 0.80 \\
\hline \multicolumn{5}{|l|}{ Insulin user } \\
\hline No & 176,878 & 47 & 1 (Ref.) & \\
\hline Yes & 72,360 & 44 & $1.19(0.63-2.25)$ & 0.59 \\
\hline \multicolumn{5}{|l|}{ Metformin user } \\
\hline No & 184,070 & 46 & 1 (Ref.) & \\
\hline Yes & 65,168 & 45 & $1.93(1.21-3.08)$ & 0.006 \\
\hline DPP4i x Insulin & & & $0.65(0.29-1.50)$ & 0.31 \\
\hline
\end{tabular}

Abbreviations: DPP4i: dipeptidyl peptidase-4 inhibitor; BP: bullous pemphigoid; HR: hazard ratio; CI: confidence interval

${ }^{a}$ Multivariable analysis was by Modified Cox proportional hazard model. Adjusted for covariate factors, including use of DPP4i, age, sex, duration of diabetes, coronary artery disease, hypertension, hyperlipidemia, renal disease, chronic liver disease and cirrhosis, chronic obstructive pulmonary 


\section{Figures}

3,034,956 DM patients between January 1, 2000, and December 31, 2015

$2,128,507$ have been prescribed antidiabetic drugs for at least 84 days within 3 months

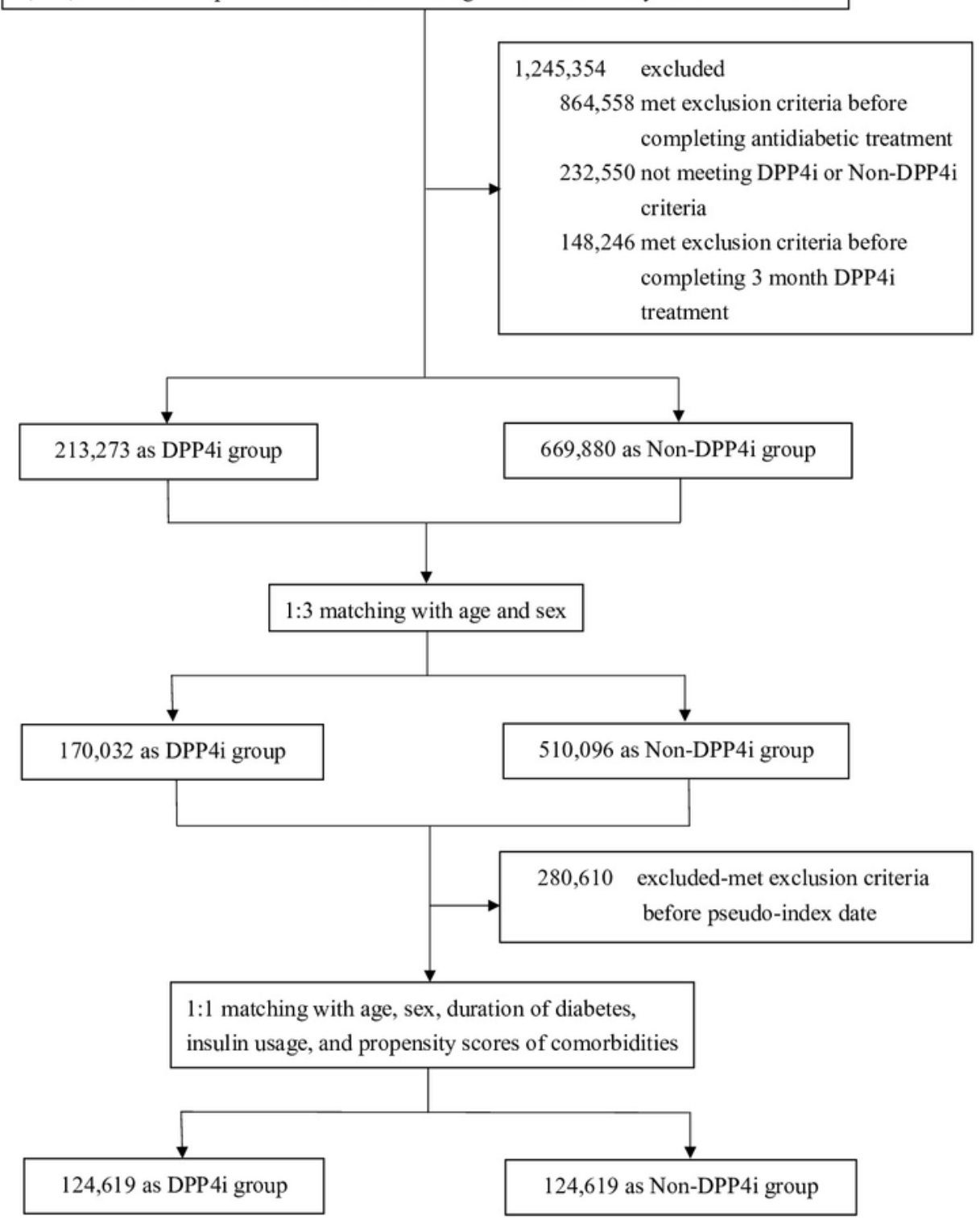

Figure 1 


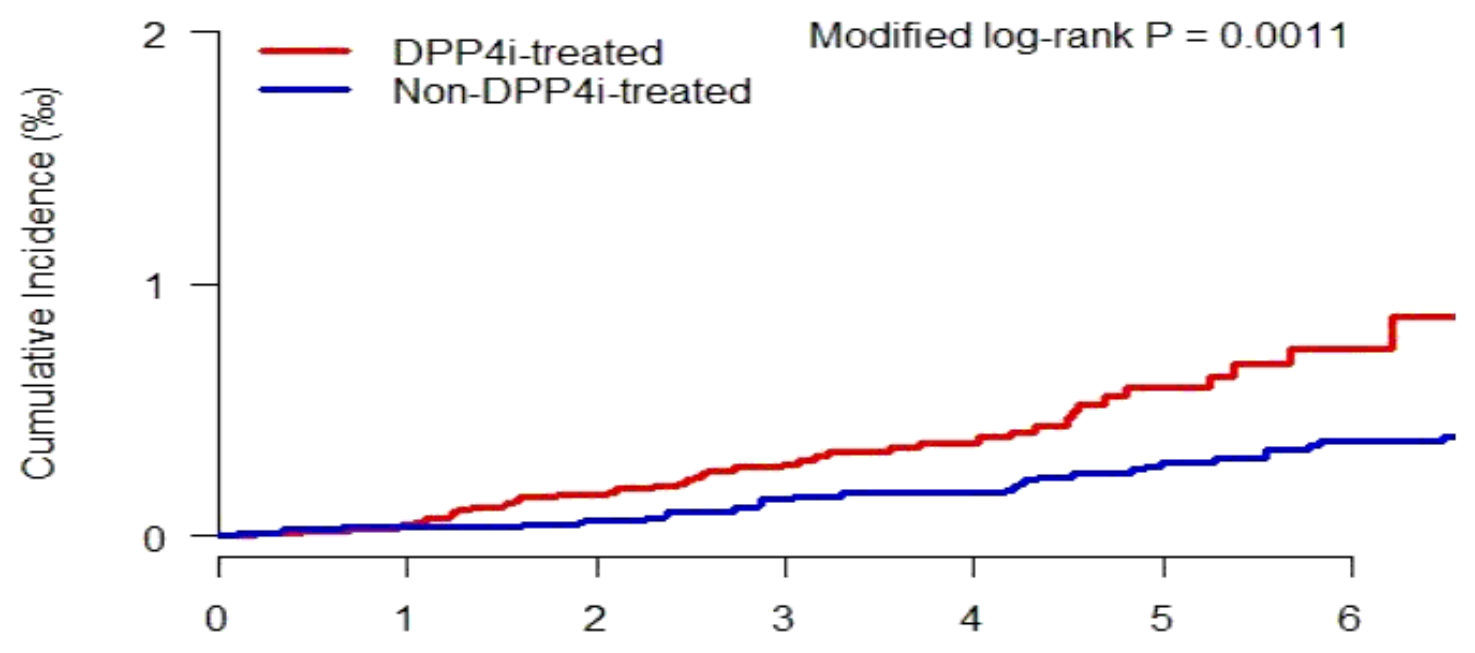

Number at risk

DPP4i-treated Non-DPP4i-treated
$124619 \quad 106031 \quad 88029$

$124619 \quad 111820 \quad 98721$

Follow up (2)

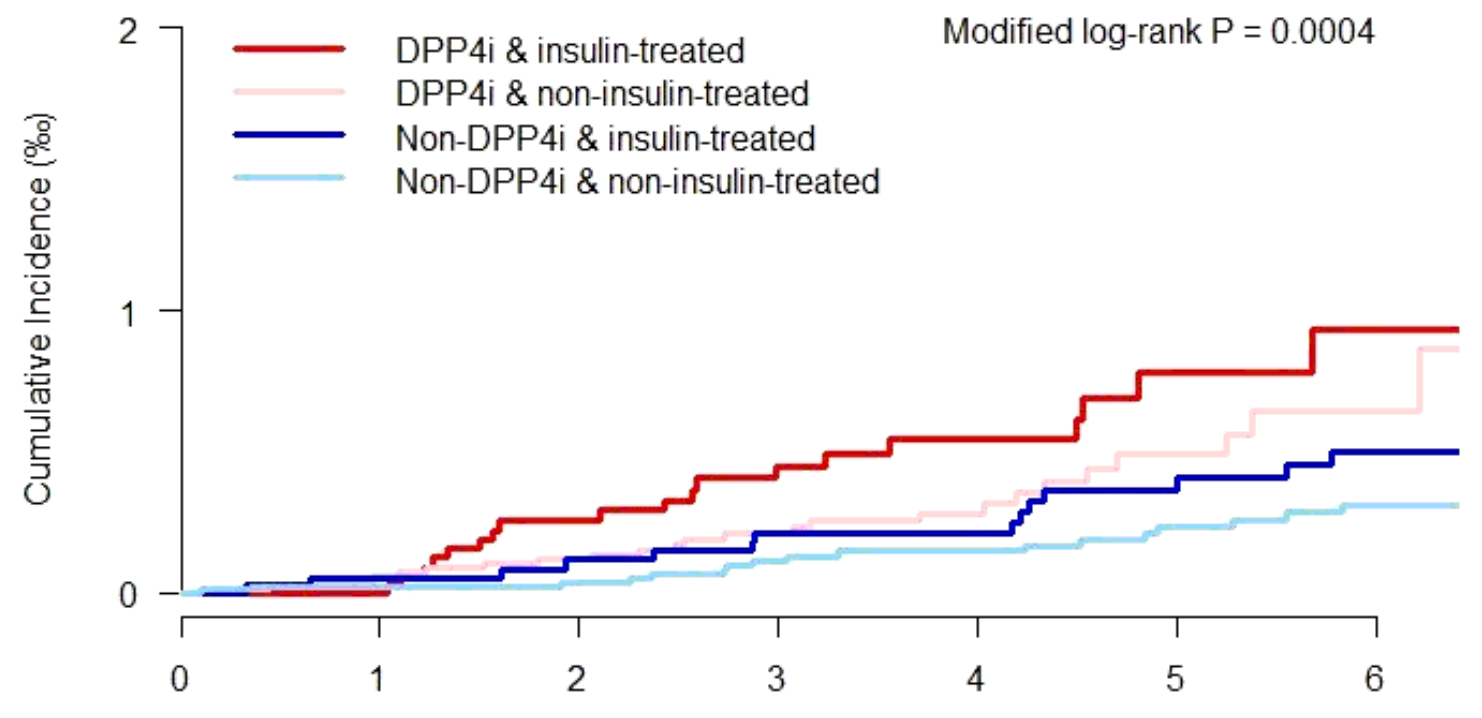

DPP4i \& insulin-treated DPP4i \& non-insulin-treated Non-DPP4i \& insulin-treated Non-DPP4i \& non-insulin-treated 88439

$\begin{array}{cc} & \text { Number at risk } \\ 36180 & 31906 \\ 88439 & 74125 \\ 36180 & 33371 \\ 88439 & 78449\end{array}$

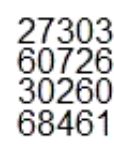

$\begin{array}{llll}68433 & 43604 & 25133 & 10084 \\ 85403 & 72096 & 59702 & 48271\end{array}$

Follow up

\section{Figure 2}

Cumulative incidences of BP for different groups by use of DPP4i or insulin. Data were compiled after adjustment for competing mortality. Cumulative incidence of BP and calculation and comparison of competing risk data ratios were determined using modified Kaplan-Meier and Gray methods.

Abbreviations: BP: bullous pemphigoid; DPP4i: dipeptidyl peptidase-4 inhibitor. 


\begin{tabular}{|c|c|c|c|c|c|}
\hline Character & \multicolumn{2}{|c|}{ DPP4i } & \multicolumn{2}{|c|}{ Non-DPP4i } & \multirow[b]{2}{*}{$\mathrm{HR}(95 \% \mathrm{Cl})$} \\
\hline Strata & $\mathbf{N}$ & D & N & D & \\
\hline \multicolumn{6}{|l|}{ Age group, y } \\
\hline$<50$ & 49035 & 7 & 49047 & 9 & $1.15(0.38-3.54)$ \\
\hline $50 \sim 59$ & 46214 & 14 & 46166 & 17 & $1.97(0.89-4.36)$ \\
\hline $60 \sim 69$ & 23260 & 15 & 23273 & 9 & $3.40(1.44-8.04)$ \\
\hline$>=70$ & 6066 & 8 & 6086 & 12 & $1.27(0.52-3.15)$ \\
\hline \multicolumn{6}{|l|}{ Sex } \\
\hline Female & 80155 & 30 & 80159 & 26 & $1.30(0.63-2.65)$ \\
\hline Male & 44420 & 14 & 44413 & 21 & $2.43(1.36-4.35)$ \\
\hline \multicolumn{6}{|c|}{ Coronary artery disease } \\
\hline Yes & 30482 & 11 & 31577 & 14 & $1.68(0.72-3.90)$ \\
\hline No & 94093 & 33 & 92995 & 33 & $1.97(1.16-3.34)$ \\
\hline \multicolumn{6}{|l|}{ Hypertention } \\
\hline Yes & 89112 & 33 & 90872 & 44 & $1.60(0.98-2.61)$ \\
\hline No & 35463 & 11 & 33700 & 3 & $5.33(1.46-19.45)$ \\
\hline \multicolumn{6}{|l|}{ Hyperlipidemia } \\
\hline Yes & 97129 & 33 & 96788 & 29 & $2.32(1.34-4.01)$ \\
\hline No & 27446 & 11 & 27784 & 18 & $1.26(0.57-2.78)$ \\
\hline \multicolumn{6}{|l|}{ Renal disease } \\
\hline Yes & 19208 & 16 & 20149 & 19 & $1.44(0.71-2.91)$ \\
\hline No & 105367 & 28 & 104423 & 28 & $2.34(1.31-4.17)$ \\
\hline \multicolumn{6}{|c|}{ Chronic liver disease and cirrhosis } \\
\hline Yes & 34472 & 9 & 37080 & 12 & $1.56(0.61-3.94)$ \\
\hline No & 90103 & 35 & 87492 & 35 & $2.00(1.20-3.34)$ \\
\hline \multicolumn{6}{|c|}{ Chronic obstructive pulmonary disease } \\
\hline Yes & 23000 & 11 & 23909 & 18 & $1.36(0.61-3.04)$ \\
\hline No & 101575 & 33 & 100663 & 29 & $2.17(1.26-3.74)$ \\
\hline \multicolumn{6}{|c|}{ Connective tissue disease } \\
\hline Yes & 3475 & 1 & 3505 & 3 & $0.64(0.06-7.03)$ \\
\hline No & 121100 & 43 & 121067 & 44 & $1.98(1.26-3.13)$ \\
\hline \multicolumn{6}{|l|}{ Insulin user } \\
\hline Yes & 36161 & 19 & 36155 & 25 & $1.56(0.82-2.96)$ \\
\hline No & 88414 & 25 & 88417 & 22 & $2.26(1.21-4.21)$ \\
\hline \multicolumn{6}{|l|}{ Metformin user } \\
\hline Yes & 34781 & 20 & 30342 & 25 & $1.67(0.88-3.15)$ \\
\hline No & 89794 & 24 & 94230 & 22 & $2.09(1.12-3.91)$ \\
\hline Overall & 124575 & 44 & 124572 & 47 & $1.75(1.14-2.67)$ \\
\hline
\end{tabular}

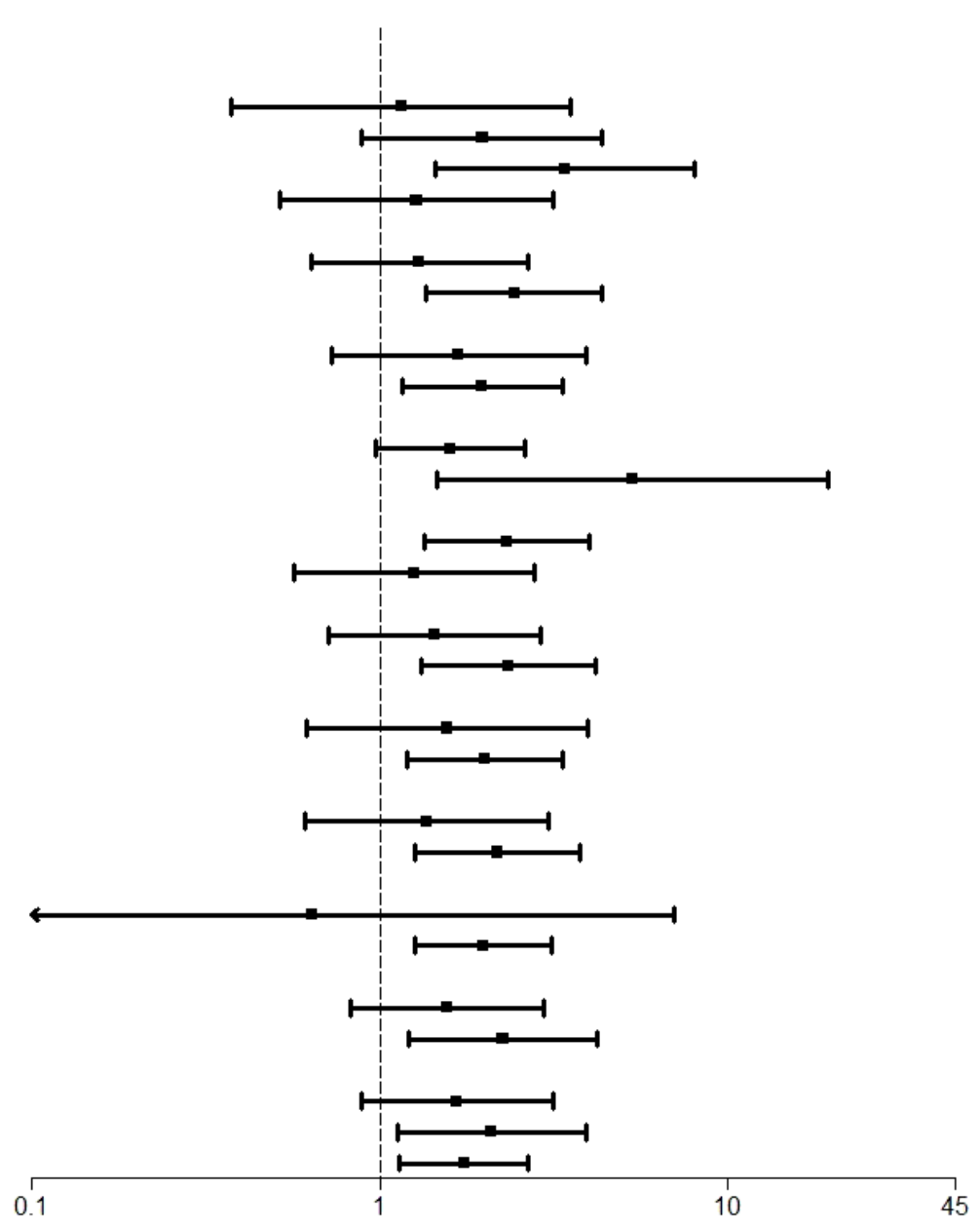

Figure 3

Multivariable stratified analyses for the association between DPP4i treatment and BP development. Multivariable analysis was by modified Cox proportional hazard model and adjusted for covariate factors, including the use of DPP4i, age, sex, duration of diabetes, coronary artery disease, hypertension, hyperlipidemia, renal disease, chronic liver disease and cirrhosis, chronic obstructive pulmonary disease, connective tissue disease, use of insulin, and use of metformin. Abbreviations: DPP4i: dipeptidyl peptidase-4 inhibitors, BP: bullous pemphigoid. 